05

\title{
Синтез тонких пленок ниобия на кремнии и исследование их сверхпроводящих свойств в области размерного кроссовера
}

\author{
() И.В. Янилкин, ${ }^{1}$ А.И. Гумаров, ${ }^{1,2}$ А.М. Рогов, ${ }^{1}$ Р.В. Юсупов, ${ }^{1}$ Л.Р. Тагиров ${ }^{2,3}$ \\ ${ }^{1}$ Казанский федеральный университет, \\ 420008 Казань, Россия \\ ${ }^{2}$ Казанский физико-технический институт им. Е.К. Завойского - \\ обособленное структурное подразделение ФИЦ КазНЦ РАН, \\ 420029 Казань, Россия \\ ${ }^{3}$ Академия наук Республики Татарстан, Институт прикладных исследований \\ 420111 Казань, Россия \\ e-mail: yanilkin-igor@yandex.ru
}

Поступило в Редакцию 19 мая 2020 г.

В окончательной редакции 13 июля 2020 г.

Принято к публикации 13 июля 2020 г.

\begin{abstract}
В условиях сверхвысокого вакуума синтезированы пленки ниобия толщиной 4-100 nm на кремниевой подложке. Измерения электрического сопротивления показали высокую температуру сверхпроводящего перехода $T_{c}$ в интервале $4.7-9.1 \mathrm{~K}$ и рекордно малые ширины переходов $\Delta T_{c}$ в интервале $260-11 \mathrm{mK}$. Исследованы зависимости $T_{c}$ и $\Delta T_{c}$ от магнитного поля и определены сверхпроводящие длины когерентности и длины свободного пробега электронов проводимости для разных толщин синтезированных пленок. Обнаружено существенное влияние магнитного поля на $\Delta T_{c}$, которое выявляет переход от трехмерной к двумерной сверхпроводимости при толщинах ниже $10 \mathrm{~nm}$. Зависимости $T_{c}$ и $\Delta T_{c}$ от толщины пленок и величины магнитного поля обсуждаются в рамках существующих теорий сверхпроводимости в тонких пленках сверхпроводящих металлов.
\end{abstract}

Ключевые слова: ниобий, тонкие пленки, сверхпроводящий переход, ширина перехода.

DOI: 10.21883/JTF.2021.02.50362.170-20

\section{Введение}

Ниобий имеет широкое применение в качестве сверхпроводника благодаря высокой $(\sim 9.2 \mathrm{~K})$ температуре сверхпроводящего (СП) перехода. Однако ниобий металл-геттер, который чрезвычайно активно реагирует, прежде всего, с кислородом, поэтому получение чистого ниобия в форме тонкой пленки для приложений в СП электронике $[1,2]$ является непростой задачей. В прикладном технологическом смысле практически невозможным оказывается получение пленки ниобия, в которой длина свободного пробега электронов проводимости $l$ превышает длину когерентности Бардина-Купера-Шриффера (БКШ) для ниобия $l>\xi_{\mathrm{BCS}}=38 \mathrm{~nm}$ [3]. Такой ниобий называют „чистым“ в терминологии физики сверхпроводников [4]. Практически же всегда выполняется обратное соотношение $l \ll \xi_{\mathrm{BCS}}$, такой ниобий называется „грязным“ [4]. Роль СП длины когерентности в этом случае выполняет величина $\xi_{s}=0.54 \sqrt{l \xi_{\mathrm{BCS}}} \ll \xi_{\mathrm{BCS}}$. Ниобий относится к группе сверхпроводников второго рода, в которые магнитное поле проникает в виде вихрей Абрикосова [4].

\section{1. Методика эксперимента}

Техника синтеза тонкопленочных структур непрерывно совершенствуется, и сегодня оказывается возмож- ным создать условия сверхвысокого вакуума, в которых осаждаемая пленка ниобия абсорбирует минимально возможное количество примесей из остаточной атмосферы вакуумной камеры с достижением максимальной температуры СП перехода $T_{c}$ для толщин $d$ в диапазоне $10-30 \mathrm{~nm}$ с очень узкими резистивными переходами в СП состояние [5-12]. В настоящей работе мы представляем результаты приготовления тонких пленок ниобия в диапазоне толщин $4-100 \mathrm{~nm}$, а также исследования параметров их переходов в СП состояние.

Синтез пленок ниобия проводился методом магнетронного распыления на монокристаллические, (100)ориентированные подложки кремния с качеством обработки поверхности уровня „ерi-ready“ (Crystal GmbH, Германия). Очистка подложек осуществлялась в многолучевой ультразвуковой ванне в ацетоне (ОСЧ) и изопропиловом спирте (ОСЧ), затем они отжигались при $800^{\circ} \mathrm{C}$ в течение $5 \mathrm{~min}$ в сверхвысоковакуумной камере (SPECS, Германия) с остаточным вакуумом $\sim 2 \cdot 10^{-8} \mathrm{~Pa}$. После отжига образец перемещался по сверхвысоковакуумной линии в камеру магнетронного распыления (BESTEC, Германия) с давлением остаточных газов после предраспыления ниобия ниже $5 \cdot 10^{-8} \mathrm{~Pa}$, в которой сначала при комнатной температуре на подложку напылялся промежуточный слой высокоомного кремния толщиной $10 \mathrm{~nm}$ (мишень 99.999\% чистоты от Гирмет, Россия). В качестве плазмообразую- 
Таблица 1. Удельное сопротивление, коэффициент остаточного сопротивления и длина свободного пробега электрона пленок ниобия различной толщины

\begin{tabular}{r|c|l|l}
\hline$d, \mathrm{~nm}$ & $\rho_{10 \mathrm{~K}}, \mu \Omega \cdot \mathrm{cm}$ & $R R R$ & $l_{\rho}, \mathrm{nm}$ \\
\hline 4 & 62 & 1.25 & 0.6 \\
5 & 53 & 1.37 & 0.71 \\
6 & 28 & 1.68 & 1.3 \\
8 & 17.7 & 2.09 & 2.1 \\
10 & 14.3 & 2.14 & 2.6 \\
15 & 12.9 & 2.45 & 2.9 \\
20 & 9.7 & 2.63 & 3.9 \\
30 & 5.8 & 3.74 & 6.4 \\
100 & 4.6 & 4.7 & 8.1
\end{tabular}

щего газа использовался аргон высокой степени очистки $(>99.9999 \%)$, при давлении $6 \cdot 10^{-1}$ Ра. Промежуточный слой свежеосажденного кремния необходим для изоляции от окисленной поверхности кремния пленки ниобия, крайне чувствительной к примесям (см. пример ухудшения свойств пленки ниобия при напылении на поверхность окисленного кремния [6]). Затем напылялся слой ниобия (мишень 99.95\% чистоты от Kurt J. Lesker, США) со скоростью $1.67 \mathrm{~nm} / \mathrm{min}$ при подаваемой на магнетрон мощности $50 \mathrm{~W}$. Температура подложки при этом составляла $120^{\circ} \mathrm{C}$ и поддерживалась автоматически контроллером нагревателя манипулятора. Затем осаждался слой кремния в $10 \mathrm{~nm}$ для защиты пленки ниобия от деградации на воздухе.

Морфология поверхности образцов исследовалась методом атомно-силовой микроскопии на приборе Bruker Dimension FastScan. Синтезированные образцы имели шероховатость $R_{q}=0.24 \mathrm{~nm}$, идентичную шероховатости подложек, что свидетельствует о конформном покрытии поверхности подложки составляющими образца. Было синтезировано 9 образцов $\mathrm{Si} / \mathrm{Nb} / \mathrm{Si}$ с различной толщиной $(d)$ слоя ниобия: $4,5,6,8,10,15,20$, $30,100 \mathrm{~nm}$. Измерение электрического сопротивления производилось стандартным 4-х контактным методом на установке PPMS-9 (Quantum Design). Алюминиевые контакты (диаметр проволоки $25 \mu \mathrm{m}$ ) присоединялись к образцам методом ультразвуковой клиновой микросварки проволочных выводов на расстоянии $0.2 \mathrm{~mm}$.

Параметры, характеризующие электропроводность полученных пленок ниобия в нормальном состоянии, удельное сопротивление при $10 \mathrm{~K} \rho_{10 \mathrm{~K}}$, коэффициент остаточного сопротивления $R R R=R_{300 \mathrm{~K}} / R_{10 \mathrm{~K}}$ и длина свободного пробега электрона, определенная по величине $\rho_{10 \mathrm{~K}},-$ представлены в табл. 1.

\section{2. Результаты и их обсуждение}

Кривые резистивных переходов для трех характерных образцов приведены на рис. 1, $a-c$. Температура СП перехода $T_{c}$ определялась на уровне $50 \%$ от сопро-
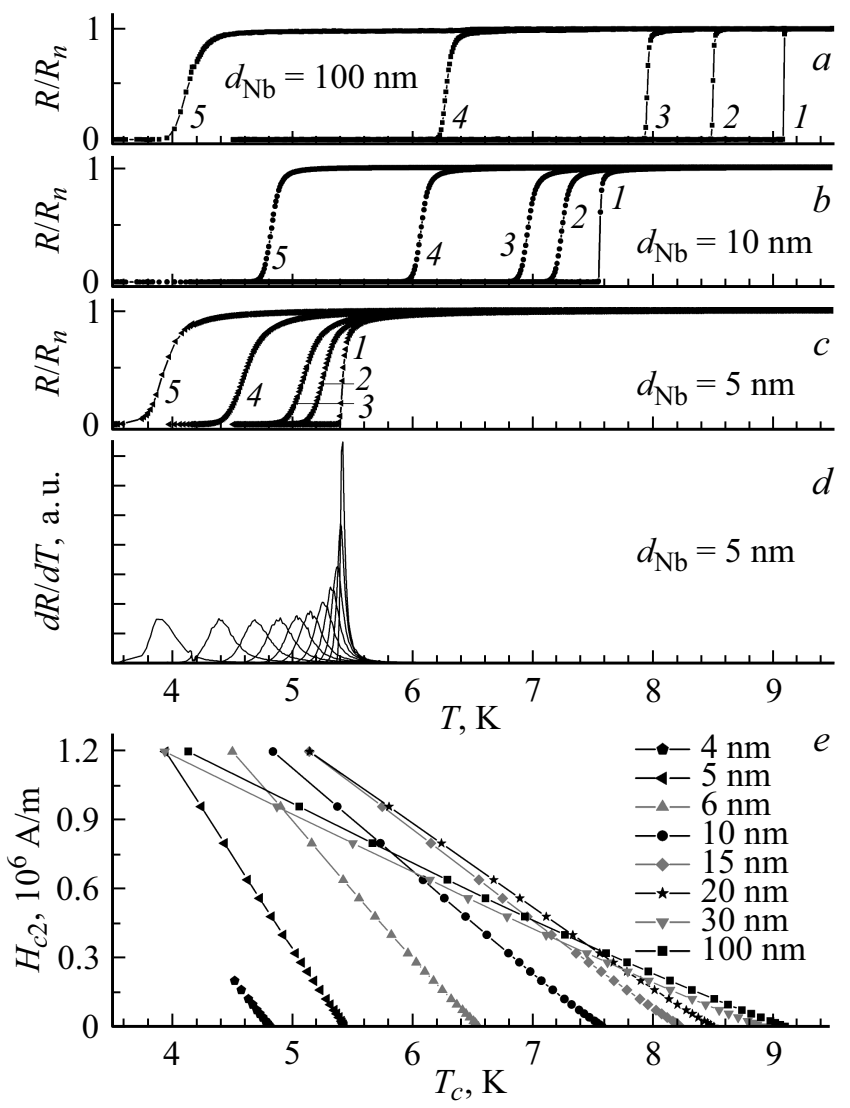

Рис. 1. $a-c-$ зависимости сопротивления пленок ниобия разной толщины $(5,10$ и $100 \mathrm{~nm}$ соответственно) от температуры в приложенном магнитном поле: $1-0,2-119.4$, 3 - 238.7, $4-636.6,5-1193.6 \mathrm{kA} / \mathrm{m} ; d-$ производные сопротивления по температуре для пленки толщиной $5 \mathrm{~nm}$; $e$ - зависимость верхнего критического поля от температуры, $H_{c 2}(T)$, для пленок ниобия полного набора толщин (кроме $8 \mathrm{~nm}$ ).

тивления $R_{n}$ в нормальном состоянии на плато выше температуры СП перехода как без магнитного поля, так и с магнитным полем (до $1.2 \mathrm{MA} / \mathrm{m}$ ), приложенным перпендикулярно плоскости пленки. Полученная таким образом зависимость верхнего критического поля $H_{c 2}$ от температуры приведена на рис. 1,e. Ширина СП перехода определялась по критерию „10-90“ как $\Delta T_{c}=T_{1}\left(0.9 R_{n}\right)-T_{2}\left(0.1 R_{n}\right)$.

Основные параметры, характеризующие СП состояние исследованной серии пленок ниобия, представлены в табл. 2. Длина когерентности в плоскости пленки $\xi_{G L 0}$ при нулевой температуре в рамках теории ГинзбургаЛандау определялась из наклона зависимостей $H_{c 2}(T)$ на рис. 1, е по формуле [4]:

$$
\xi_{G L 0}=\left[-\frac{2 \pi T_{c 0}}{\Phi_{0}} \frac{d H_{c 2 \perp}(T)}{d T}\right]^{-1 / 2},
$$

где $\Phi_{0}-$ квант магнитного потока, $H_{c 2}-$ величина верхнего критического поля, $T_{c 0}-$ критическая тем- 
Таблица 2. Параметры, характеризующие переходы и сверхпроводящее состояние пленок ниобия различной толщины

\begin{tabular}{r|c|c|c|c|c|c}
\hline$d, \mathrm{~nm}$ & $T_{c}, \mathrm{~K}$ & $\Delta T_{c}, \mathrm{~K}$ & $H_{c 20}, \mathrm{MA} / \mathrm{m}$ & $\xi_{G L 0}, \mathrm{~nm}$ & $\xi_{S}, \mathrm{~nm}$ & $l_{\xi}, \mathrm{nm}$ \\
\hline 4 & 4.72 & 0.261 & & & & \\
5 & 5.43 & 0.188 & 4.43 & 7.8 & 5.0 & 2.2 \\
6 & 6.54 & 0.096 & 3.79 & 8.7 & 5.5 & 2.7 \\
8 & 7.34 & 0.047 & & & & \\
10 & 7.57 & 0.044 & 3.3 & 9.4 & 6.0 & 3.2 \\
15 & 8.22 & 0.026 & 3.17 & 9.45 & 6.0 & 3.2 \\
20 & 8.49 & 0.025 & 3.0 & 9.3 & 5.9 & 3.1 \\
30 & 8.90 & 0.021 & 2.16 & 11.8 & 7.5 & 5 \\
100 & 9.08 & 0.011 & 2.15 & 11.8 & 7.5 & 5
\end{tabular}

пература в отсутствие магнитного поля. Сверхпроводящая длина когерентности $\xi_{s}=0.54 \sqrt{l \xi_{\mathrm{BCS}}}$ находится из $\xi_{G L 0}$ с помощью соотношения $\xi_{S}=(2 / \pi) \xi_{G L 0}$, что дает возможность оценить длину свободного пробега электронов проводимости $l_{\xi}$ и ее зависимость от толщины. Из табл. 2 видно, во-первых, что ниобий в наших пленках является „грязным“ сверхпроводником с $l_{\xi} \ll \xi_{\mathrm{BCS}}(\mathrm{Nb})=38 \mathrm{~nm}$. Во-вторых, стоит отметить, что синтезированные нами пленки ниобия на кремниевой подложке имеют самые высокие $T_{c}$ из опубликованных в литературе [6-12] и характеризуются малой шириной переходов в нулевом поле, что свидетельствует о высоком качестве полученных образцов.

Исследование СП переходов в приложенном перпендикулярном магнитном поле (рис. 1 и табл. 2) показывает, что температура СП перехода падает как с уменьшением толщины пленки, так и с ростом напряженности поля. Подавление $T_{c}$ при приближении толщины пленки СП металла $d$ к длине когерентности $\xi_{S}$ происходит вследствие нескольких причин. Основной принято считать усиление кулоновского отталкивания между электронами, входящими в объем, определяемый длиной когерентности $\xi_{S}$, из-за малой длины свободного пробега, составляющей 1-2 nm для самых тонких пленок (табл. 1 и 2) [13]. Вследствие усиления кулоновского взаимодействия растет и спиновая восприимчивость электронов [14], что также не способствует сверхпроводимости, однако в значительно меньшей степени. Влияние прямого контакта с кремниевыми обкладками пленки (напомним, что подстилающий и укрывающий слои кремния имеют толщину $\sim 10 \mathrm{~nm}$ ) может внести свой вклад в понижение $T_{c}$. Несмотря на то, что при температуре около $5 \mathrm{~K}$ высокоомный при комнатной температуре кремний является изолятором, он может перенормировать спектр фононов тонкого по сравнению с ним ниобия $(10 \mathrm{~nm}$ и ниже). Действительно, длина волны даже дебаевских фононов ниобия $\left(T_{\text {Debye }}=276 \mathrm{~K}\right)$ значительно превышает толщину пленки, поэтому перпендикулярные плоскости пленки фононы ниобия не могут остаться независимыми. Однако мы не ожидаем значительного эффекта от перенормировки фононов и его сильной зависимости от толщины. А вот квантовые размерные эффекты на малых толщинах могут дать существенный вклад, так как пленка ниобия толщиной $4 \mathrm{~nm}$ - это всего 12 монослоев. Поэтому для тонкой пленки спектр электронных состояний дискретен в направлении нормали к ее плоскости, и в уравнении БКШ для параметра порядка должно проводиться суммирование по считанному числу разрешенных волновых векторов в этом направлении. $3 D-2 D$-переход может вносить доминирующий вклад на малых толщинах, так как предельный случай предсказуем - в чисто двумерной системе (монослой) теорема Мермина-Вагнера запрещает дальний порядок [15].

С ростом магнитного поля $T_{c}$ также понижается, при этом эффективность подавления СП состояния зависит от толщины пленки: чем толще пленка, тем быстрее падает температура перехода в магнитном поле. Это согласуется с теорией Маки-Ларкина [16,17], в которой был предложен механизм разрушения куперовских пар, обусловленный влиянием магнитного поля на орбитальное движение электронов. Параметром разрушения пар является величина $\alpha=D e H / 2 \pi T_{c}$, в которой $D=l v_{\mathrm{F}} / 3$ есть коэффициент диффузии электронов проводимости, $e, v_{\mathrm{F}}$ и $l-$ заряд, фермиевская скорость и длина свободного пробега электронов проводимости, а $H-$ напряженность магнитного поля (сверхпроводник считается грязным, а магнитное поле близким к верхнему критическому, что соответствует условиям наших измерений). Выраженный через длину когерентности (табл. 2, шестой столбец), $\alpha \propto\left(\xi_{S}\right)^{2}$, с уменьшением толщины пленки параметр разрушения пар уменьшается, а верхнее критическое поле (нелинейно) увеличивается. Соответственно при одинаковом значении поля $T_{c}$ в тонкой пленке подавляется значительно слабее, чем в толстой, что мы и видим на рис. $1, a-c, e)$.

Зависимости ширины резистивного СП перехода $\Delta T_{c}$ от величины магнитного поля для исследованной серии пленок ниобия показаны на рис. 2, $а$. Видно, что характер этих зависимостей качественно изменяется в пределах серии. Для толстых пленок (30-100 nm) наблюдается небольшой (до $50 \mathrm{mK}$ ) резкий рост ширины перехода в малых полях до $24 \mathrm{kA} / \mathrm{m}$, который сменяется плавным монотонным увеличением с дальнейшим ростом поля. Для средних толщин $(10-20 \mathrm{~nm})$ рост $\Delta T_{c}$ с большим инкрементом происходит в более широкой области величин магнитного поля (до $160 \mathrm{kA} / \mathrm{m}$ ), и само $\Delta T_{c}$ существенно больше - от 90 до $170 \mathrm{mK}$, в зависимости от толщины пленки ниобия. Затем при дальнейшем увеличением напряженности приложенного поля, $\Delta T_{c}(H)$ остается практически неизменной. Для тонких пленок $(4-6 \mathrm{~nm})$ начальный рост ширины перехода происходит растянуто до еще больших полей $(\sim 560 \mathrm{kA} / \mathrm{m}$ при $5 \mathrm{~nm}$ ), сам переход становится широким с тенденцией для $\Delta T_{c}(H)$ уменьшаться с ростом величины магнитного поля $H$. Обзор формы кривых резистивного перехода на рис. 1 позволяет предположить, что значи- 


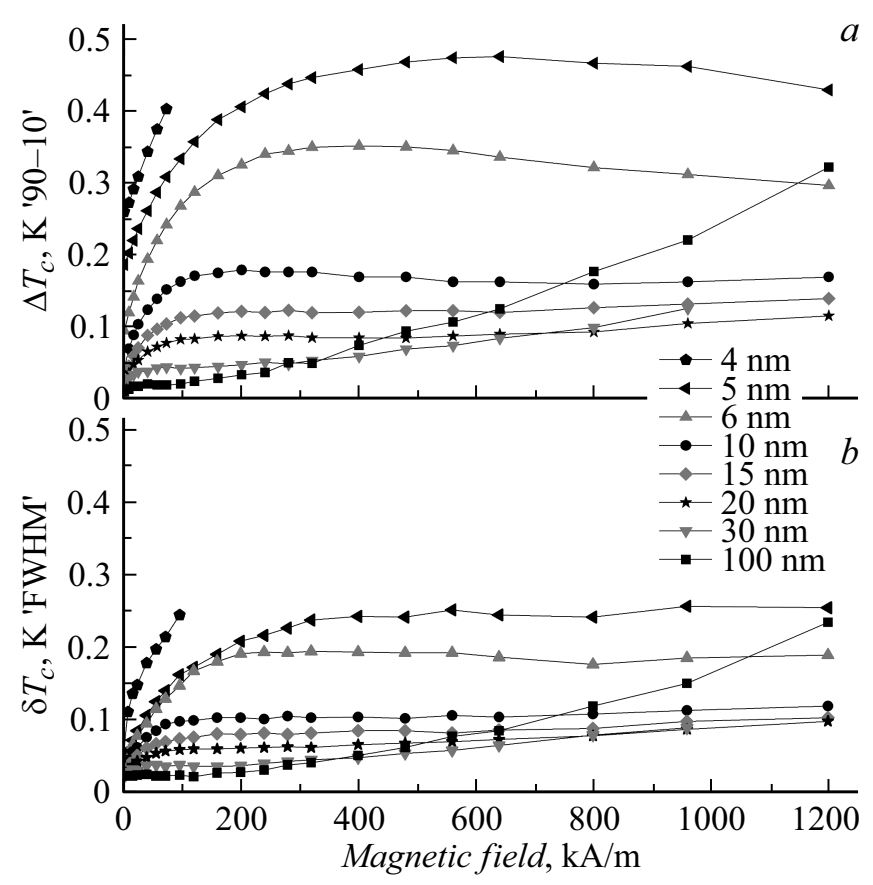

Рис. 2. Зависимости ширины СП перехода от величины перпендикулярного поверхности магнитного поля для пленок ниобия различной толщины: $a-$ ширина $\Delta T_{c}(H)$ определена по критерию „10-90“, $b-\delta T_{c}(H)$ определена по ширине пика производной $d R(T, H) / d T$ на ее полувысоте. Линии соединяют точки.

тельное увеличение ширины СП перехода происходит за счет сглаживания его высокотемпературного плеча.

Чтобы проверить это предположение количественно, мы обработали экспериментальные данные, используя другой критерий определения температуры СП перехода - по максимуму производной $d R(T, H) / d T$, а ширины СП перехода - по ширине линии производной $d R(T, H) / d T$ на ее полувысоте $-\delta T_{c}(H)$. Для пленки толщиной $5 \mathrm{~nm}$ производные переходов по температуре показаны на рис. $1, d$. Построение зависимости $H_{c 2 \perp}(T)$ с использованием положения максимума производной дает зависимости, практически идентичные таковым на рис. 1,e с максимальным относительным отличием в $3.5 \%$ для толщины пленки $5 \mathrm{~nm}$ и практически полным совпадением, начиная с $10 \mathrm{~nm}$ в сторону увеличения толщины. Таким образом, $T_{c}\left(H, d_{\mathrm{Nb}}\right)$, определяемые по обоим критериям, практически одинаковы в пределах погрешности измерений.

Зависимости ширины СП перехода $\Delta T_{c}(H)$ от магнитного поля для всех толщин пленок с использованием $d R(T, H) / d T$ собраны на рис. $2, b$. Результаты построения разительно отличаются от таковых для критерия „10-90“. Сравнение рис. $2, a$ и $b$ позволяет заключить следующее:

1) $\delta T_{c}(H)$ практически вдвое меньше $\Delta T_{c}(H)$, что формально следует из того, что большая часть высоко- температурного плеча перехода находится за пределами ширины пика производной $d R(T, H) / d T$;

2) в зависимости $\Delta T_{c}(H)$ немонотонность, просматривающаяся на рис. 2, а для толщин пленки в 5, 6, и $10 \mathrm{~nm}$, пропадает в пределах погрешности измерений (для убедительности аргументации мы не применяли никакой предварительной обработки кривых производной, как сглаживание или моделирование).

Таким образом, анализ зависимости ширины перехода от магнитного поля позволяет нам сделать следующие выводы:

a) при уменьшении толщины пленок до величины СП длины когерентности и ниже, ширины переходов $\Delta T_{c}(H)$ и $\delta T_{c}(H)$ по обоим критериям резко возрастают, отражая переход от трехмерного $(3 D)$ к двумерному $(2 D)$ режиму сверхпроводимости;

б) для тонких пленок $(4,5,6 \mathrm{~nm})$ примерно половину вклада в ширину перехода $\Delta T_{c}(H)$ по критерию „10-90“ и ее немонотонность вносит высокотемпературное плечо резистивного перехода, которое также подвержено значительному влиянию изменения размерности сверхпроводимости.

Как и в случае $T_{c}$, на поведение ширины СП перехода оказывает влияние ряд причин: это статические и динамические флуктуации. Вариации толщины пленки на больших масштабах (мы можем допустить „завал“ толщины в 1-2\% от края до края образца), градиент совершенства решетки (кристалличности [18] и содержания примесей - СП свойства улучшаются с ростом толщины пленки, в частности, и потому, что распыляемый ниобий как геттер „чистит“ остаточную атмосферу камеры в процессе осаждения ниобиевой пленки) могут приводить к затянутому переходу со стороны температур выше $T_{c}$, тогда как мелкомасштабные (шероховатость, обусловленная морфологией поверхности подложки и подстилающего слоя кремния на ней) может влиять на силу пиннинга вихрей Абрикосова при их входе в образец при СП переходе. Это также приводит к затягиванию перехода, однако, ожидаемому на низкотемпературной „ступне“ перехода $[5,18]$. Рис. $1, d$ показывает, что этот механизм вносит определенный вклад в ширину перехода $\delta T_{c}(H)$ и далее в $\Delta T_{c}(H)$. Кроме того, со стороны „высокотемпературного“ плеча ожидается существенный вклад динамических флуктуаций (Асламазова-Ларкина и Маки-Томсона [19-24]) избыточной проводимости, обусловленной рождением куперовских пар выше $T_{c}$ и их разрушением температурой. С уменьшением толщины пленки и переходом от $3 D$ к $2 D$ сверхпроводимости совершенно естественно ожидать, что флуктуации проявятся на большем интервале выше $T_{c}$ и их амплитуда будет больше, так как ограничение двумя измерениями препятствует установлению стабильного дальнего порядка.

Качественные различия в ходе зависимостей ширины СП перехода от магнитного поля (рис. 2) могут быть связаны с влиянием динамических флуктуаций [19-24] 
и поверхностной сверхпроводимости [25]. Определенно, поверхностной сверхпроводимостью можно объяснить монотонный рост ширины перехода по критерию „10-90“ (рис. 2, a) для пленки толщиной $100 \mathrm{~nm}$, в которой при длине когерентности порядка $10 \mathrm{~nm}$ достаточно объема для развития поверхностной сверхпроводимости на границах кристаллитов [25]. На это указывает и последовательное падение инкремента роста ширины перехода до малых величин в пленках толщиной 30 и $20 \mathrm{~nm}$ в интересующем нас диапазоне приложенных полей. В пленках толщиной $4-15 \mathrm{~nm}$, представляющих для нас наибольший интерес, по-видимому, нет пространства для развития поверхностной сверхпроводимости. Величина же флуктуационного вклада в проводимость зависит не только от „отдаленности“ текущей температуры от критической, определяемой параметром $\varepsilon=\left(T-T_{c}\right) / T_{c}$, но и от абсолютной температуры. Понятно, что термическая энергия, разрушающая флуктуационные пары при $3 \mathrm{~K}$, будет в два раза меньше таковой при $6 \mathrm{~K}$, поэтому при понижении абсолютной температуры перехода с ростом поля скорость распада пар падает, и флуктуационный вклад уменьшается, приводя к немонотонному поведению $\Delta T_{c}(H)$ с ростом поля, что наблюдается в нашем эксперименте. Доминирующим вкладом в $\delta T_{c}(H)$ на малых толщинах пленок, скорее всего, являются статические и динамические неоднородности.

Какое определение ширины перехода следует использовать зависит от области применения тонких пленок ниобия. Для джозефсоновской физики ширина перехода в разумных пределах не важна вообще, так как большинство измерений и приложений проводятся при температурах намного ниже $T_{c}$. Если фокусом исследования является сдвиг $T_{c}$, то при автоматизированном измерении сопротивления ее $\left(T_{c}\right)$ практически автоматически можно определять из положения максимума производной $d R(T, H) / d T$. Если исследуются переключающие свойства гетероструктуры с ниобиевыми слоями [26-28], то влияние управляющей магнитной субструктуры на $T_{c}$ должно превышать ширину перехода $\Delta T_{c}(\mathrm{H})$ по критерию „10-90“, чтобы получить полное переключение между нормальным и СП состояниями.

\section{Заключение}

Таким образом, в широком диапазоне толщин пленок ниобия была исследована зависимость температуры СП перехода $T_{c}$ и его ширины от толщины пленок и величины магнитного поля. Экспериментально получены очень узкие переходы с наиболее высокой $T_{c}$ из опубликованных для пленок на кремниевой подложке. Зависимости $T_{c}$ и ширины СП перехода от толщины пленок и величины магнитного поля обсуждены в рамках существующих теоретических представлений.

\section{Финансирование работы}

Работа выполнена при финансовой поддержке РФФИ (проект № 18-32-01041 мол_а) с использованием оборудования ФЦКП ФХИ КФУ и Междисциплинарного центра аналитической микроскопии КФУ. Работа Л.Р. Тагирова была поддержана государственным заданием ФИЦ КазНЦ РАН № AААА-А18-118030690040-8.

\section{Конфликт интересов}

Авторы заявляют, что у них нет конфликта интересов.

\section{Список литературы}

[1] S.K. Tolpygo. Low Temperature Phys., 42, 361 (2016). DOI: $10.1063 / 1.4948618$

[2] I.I. Soloviev, N.V. Klenov, S.V. Bakurskiy, M.Yu. Kupriyanov, A.L. Gudkov, A.S. Sidorenko. Beilstein J. Nanotechnol., 8, 2689 (2017). DOI: $10.3762 /$ bjnano.8.269

[3] Ch. Kittel. Introduction to Solid State Physics (Seventh edition, John Wiley \& Sons, Inc., 1996).

[4] M. Tinkham. Introduction to Superconductivity (2nd ed. McGraw-Hill, NY., 1996).

[5] D. Stamopoulos, A. Speliotis, D. Niarchos. Supercond. Sci.Technol., 17 (11), 1261 (2004). DOI: $10.1088 / 0953-2048 / 17 / 11 / 006$

[6] N. Pinto, S.J. Rezvani, A. Perali, L. Flammia, M.V. Milošević, M. Fretto, C. Cassiago, N. De Leo. Sci. Rep., 8 (1), 1 (2018). DOI: 10.1038/s41598-018-22983-6

[7] A.S. Sidorenko, V.I. Zdravkov, A. Prepelitsa, C. Helbig, Y. Luo, S. Gsell, M. Schreck, S. Klimm, S. Horn, L.R. Tagirov, R. Tidecks. Annalen der Phys. (Leipzig-Berlin), 12 (1-2), 37 (2003). DOI: 10.1002/andp.200310005

[8] A. Gubin, K. Il'in, S. Vitusevich, M. Siegel, N. Klei. Phys. Rev. B, 72, 064503-8 (2005). DOI: 10.1103/PhysRevB.72.064503

[9] V. Zdravkov, A. Sidorenko, G. Obermeier, S. Gsell, M. Schreck, C. Müller, S. Horn, R. Tidecks, L.R. Tagirov. Phys. Rev. Lett., 97 (5), 057004 (2006).

DOI: 10.1103/PhysRevLett.97.057004

[10] C. Cirillo, A. Rusanov, C. Bell, J. Aarts. Phys. Rev. B, 75, 174510 (2007). DOI: 10.1103/PhysRevB.75.174510

[11] Th.R. Lemberger, I. Hetel, J.W. Knepper, F.Y. Yang. Phys. Rev. B, 76, 094515 (2007). DOI: 10.1103/PhysRevB.76.094515

[12] V.I. Zdravkov, J. Kehrle, G. Obermeier, S. Gsell, M. Schreck, C. Müller, H.-A. Krug von Nidda, J. Lindner, J. MoosburgerWill, E. Nold, R. Morari, V.V. Ryazanov, A.S. Sidorenko, S. Horn, R. Tidecks, L.R. Tagirov. Phys. Rev. B, 82 (21), 054517 (2010). DOI: 10.1103/PhysRevB.82.054517

[13] A.M. Finkel'stein. Physica B, 197, 636 (1994). DOI: $10.1016 / 0921-4526(94) 90267-4$

[14] A.M. Finkel'stein. J. de Phys., 49, 1173 (1988). DOI: $10.1051 /$ jphyscol:19888539

[15] N.D. Mermin, H. Wagner. Phys. Rev. Lett., 17, 1133 (1966). DOI: 10.1103/PhysRevLett.17.1133

[16] K. Maki. Physics, 1 (1), 21 (1964). DOI: 10.1103 /PhysicsPhysiqueFizika.1.21

[17] A.I. Larkin. Sov. Phys. JETP, 21 (1), 153 (1965).

[18] C. Delacour, L. Ortega, M. Faucher, T. Crozes, T. Fournier, B. Pannetier, V. Bouchiat. Phys. Rev. B, 83, 144504 (2011). DOI: 10.1103/PhysRevB.83.144504 
[19] L.G. Aslamazov, A.I. Larkin. Sov. Phys. Solid State, 10 (4), 875 (1968).

[20] K. Maki. Prog. Theor. Phys., 40 (2), 193 (1968). DOI: 10.1143/PTP.40.193

[21] S. Thompson. Phys. Rev. B, 1, 327 (1970). DOI: 10.1103/PhysRevB.1.327

[22] M.Yu. Reizer. Phys. Rev. B, 45, 12949 (1992). DOI: 10.1103/PhysRevB.45.12949

[23] M.H. Theunissen, P.H. Kes. Phys. Rev. B, 55, 15183 (1997). DOI: 10.1103/PhysRevB.55.15183

[24] A.I. Larkin, A.A. Varlamov. Fluctuation Phenomena in Superconductors. In: Superconductivity. Conventional and Unconventional Superconductors (ed. by K.H. Bennemann, J.B. Ketterson, Springer-Verlag Berlin Heidelberg, 2008), ch. 10 .

[25] A. Zeinali, T. Golod, V.M. Krasnov. Phys. Rev. B, 94, 214506 (2016). DOI: 10.1103/PhysRevB.94.214506

[26] P.V. Leksin, N.N. Garifyanov, I.A. Garifullin, J. Schumann, H. Vinzelberg, V. Kataev, R. Klingeler, O.G. Schmidt, B. Büchner. Appl. Phys. Lett., 97, 102505 (2010). DOI: $10.1063 / 1.3486687$

[27] V.I. Zdravkov, D. Lenk, R. Morari, A. Ullrich, G. Obermeier, C. Müller, H.A. Krug von Nidda, A.S. Sidorenko, S. Horn, R. Tidecks, L.R. Tagirov. Appl. Phys. Lett., 103, 062604 (2013). DOI: 10.1063/1.4818266

[28] D. Lenk, R. Morari, V.I. Zdravkov, A. Ullrich, G. Obermeier, C. Müller, A.S. Sidorenko, H.A. Krug von Nidda, S. Horn, L.R. Tagirov, R. Tidecks. Phys. Rev. B, 96, 184521 (2017). DOI: 10.1103/PhysRevB.96.184521 